\title{
Post-operative nausea and vomiting in bariatric patients undergoing laparoscopic sleeve gastrectomy
}

\author{
H.-J. Cho' ${ }^{1}$, Y.-H. Huang ${ }^{1}$, K.-S. Poon ${ }^{1}$, K.-B. Chen ${ }^{1}$, M.-S. Lee ${ }^{2}$, K. H. Liao' ${ }^{1}$ \\ 1. Department of Anesthesiology, China Medical University Hospital - Taichung (Taiwan) \\ 2. Department of Surgery, Division of Bariatric Surgery, China Medical University Hospital - Taichung (Taiwan)
}

\section{Background}

Studies have shown the incidence of postoperative nausea and vomiting (PONV) in patients undergoing bariatric surgeries was very high in spite of pharmacological prophylaxis or adjustment of anesthetics. Nevertheless, evidence has indicated goal-directed fluid therapy (GDFT) improves outcomes including PONV in major abdominal surgeries. The aim of our study is to investigate the effect of GDFT on reducing the incidence of PONV in patients undergoing laparoscopic sleeve gastrectomy (LSG).

\section{Material and Methods}

\section{Patient's enrollment:}

1. $\mathrm{BMI}>40$, or $\mathrm{BMI}>35$ with metabolic syndromes

2. NPO for 8 hours

3. Exclusion criteria were age $<20$ or $>65$ years-old, or comorbid with major organs' dysfunction

Participants were randomized to 3 groups: control group, GDFT-HES (GH) group and GDFT-Lactate Ringer (GL) group. Study protocol:

An non-invasive hemodynamic monitor (ClearSight ${ }^{\mathrm{TM}}$ ) was applied in GDFT groups for evaluation of stroke volume variation (SVV), and the strategy of intraoperative fluid management for GDFT groups was followed as the flowchart below.

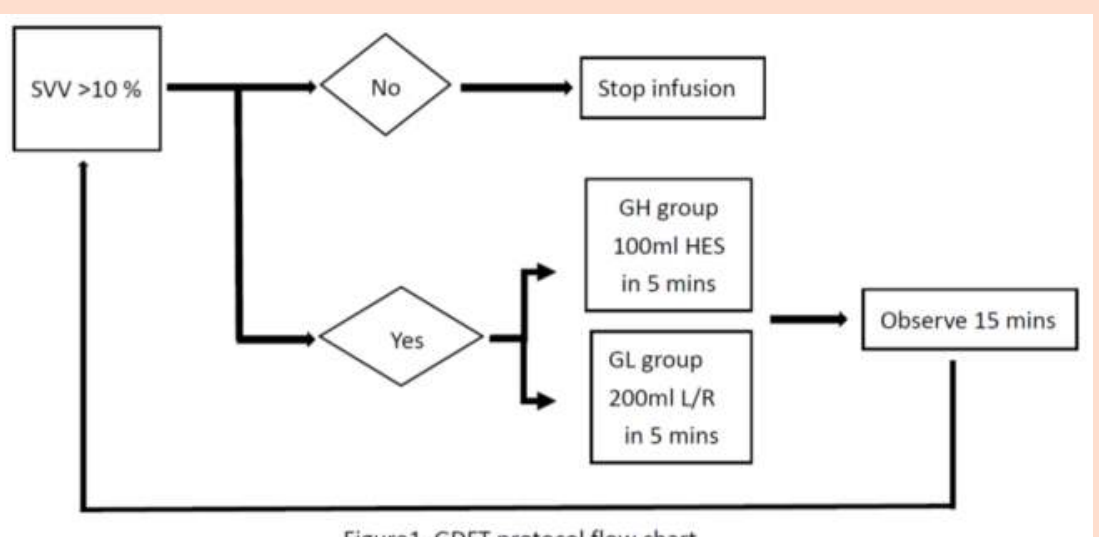

For control group, the total infused volume was lean body mass-based 4-2-1 rule for 8 h fluid deficit plus Lactate Ringer $4 \mathrm{ml} / \mathrm{kg} / \mathrm{h}$ during the surgical period. PONV was evaluated by a standardized questionnaire at $0 \mathrm{~min}, 30 \mathrm{~min}, 1 \mathrm{~h}, 24 \mathrm{~h}$ and $48 \mathrm{~h}$ after the surgery with numeric rating score from 0 to 10 .

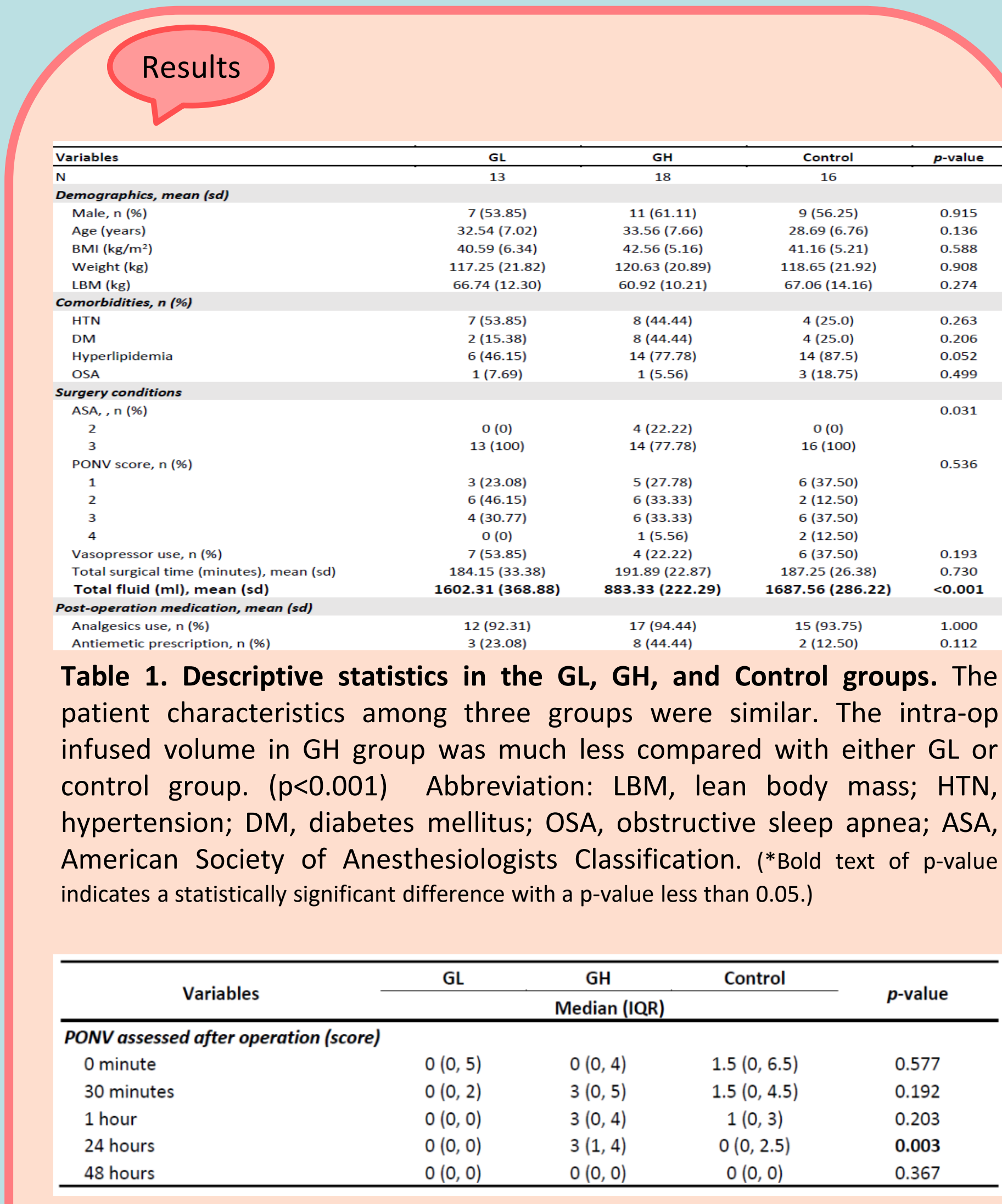

Table 2. PONV score assessment after operation 0min, 30min, 1h, 24h, and $48 \mathrm{~h}$ among $\mathrm{GL}, \mathrm{GH}$, and Control groups. Among these 3 groups, no significant difference in PONV score was observed at all time points except post-operative $24 \mathrm{~h}$.

\begin{tabular}{lccc}
\hline \multirow{2}{*}{ Group } & GL & GH & Control \\
\cline { 2 - 4 } & & $p$-value & \\
\hline GL & - & 0.001 & 0.196 \\
GH & 0.001 & - & 0.036 \\
Control & 0.196 & 0.036 & - \\
\hline
\end{tabular}

Table 3. Detail analysis following table 2 among $\mathbf{G L}, \mathbf{G H}$, and control group in PONV score assessed at post-op 24h. The data showed there was no difference between GL and control group. However, PONV score was higher in GH group compared with either GL or control group.

Discussion Surprisingly, there was no difference in intra-op infused volume between GL group and control group, thus no difference

Discussion the incidence of PONV was observed at all time points. It was speculated that LSG is a relatively hemodynamicallystable surgery without massive hemorrhage or intravascular volume shifting. The surgical duration is usually 2 to 3 hours by an experienced surgeon. The diversity of intra-op volume status will not present between GDFT and weight-based fluid strategy. It is to be noticed, on the basis of GDFT, participants in GH group were more subjected to PONV at post-op 24h compared with those in GL group. Hydroxyethyl starch (HES) had been reported the effect of degradation of endothelial glycocalyx and increase of intestinal capillary permeability. We hypothesize intra-op HES infusion would lead to disturbance of intestinal peristalsis and increase the incidence of PONV following intestinal edema caused by intestinal endothelial damage.

Summary There is no significant benefit using GDFT to decrease PONV with intra-op crystalloid infusion by using the fluid strategies in our study protocol. Modification of GDFT parameters will be needed to determine its effect in this surgical population. In addition, PONV rate will be higher with intra-op colloid infusion for GDFT during LSG because of potential pathologic jeopardy in vascular endothelium of gastrointestinal tract. Further investigation should be carried out to elucidate the mechanism. 